\title{
Supplementary
}

\section{Carnivorous Nutrition in Pitcher Plants (Nepenthes spp.) via an Unusual Complement of Endogenous Enzymes}

Linda Lee, Ye Zhang, Brittany Ozar, Christoph W. Sensen, David C. Schriemer*

\author{
Affiliations: \\ Graz University of Technology, Institute of Molecular Biotechnology, Graz, Austria \\ Christoph W. Sensen \\ Department of Biochemistry and Molecular Biology and the Southern Alberta Cancer \\ Research Institute, University of Calgary, Calgary, Alberta, Canada \\ Linda Lee, Ye Zhang, Brittany Ozar, David C. Schriemer \\ *corresponding author: D.C. Schriemer \\ Faculty of Medicine, University of Calgary, 3330 Hospital Dr. NW, Calgary, Canada, T2N 4N1. \\ Ph: 403-210-3811 email: dschriem@ucalgary.ca
}




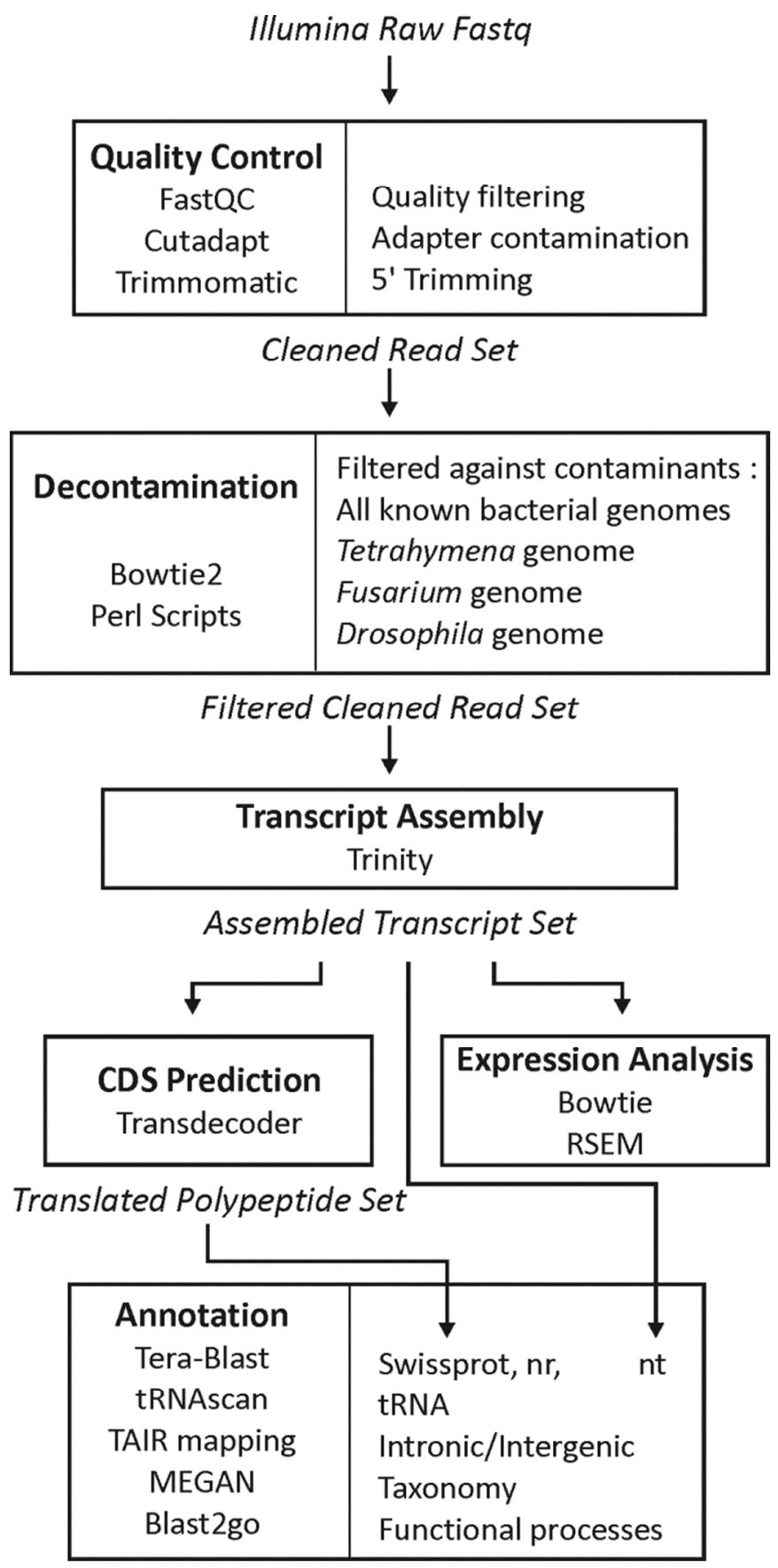

Figure S1. Data analysis for the construction of the $N$. rafflesiana transcriptome. Raw sequencing data was subjected to quality control evaluation and filtered against known adaptors and contaminant genomes. The transcriptome was assembled using Trinity and annotated using Blast. Additional annotation steps were performed to extend annotation of transcripts without homologous hits against primary databanks. MEGAN, in combination with taxonomic information gathered from nucleotide level hits against nt was used to extract a plant transcript set from the overall annotation set. An expression filtered plant transcript assembly set was used as input to Blast2Go for functional assignment. 
Nepenthesins

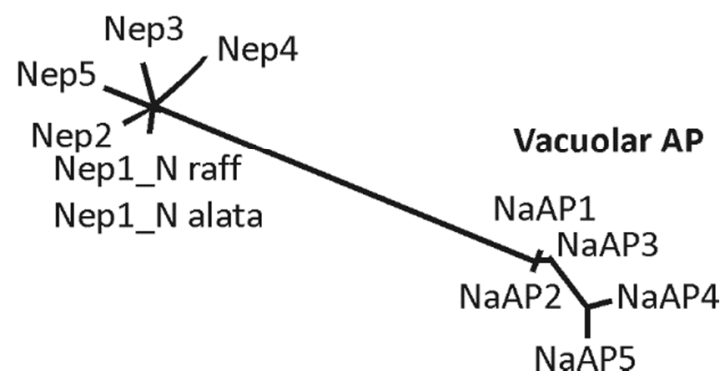

Figure S2. Phylogenic tree comparing the nepenthesins from $N$. rafflesiana ( $N$ raff) and $N$. alata Nep1 with $N$. alata vacuolar aspartic proteases (NaAP1-5) identified by An et al. ${ }^{36}$ 
List S1. Amino acid sequence of the proteins identified in N. rafflesiana digestive fluid

1. Nepenthesin-1, NrNep1

MASSLYSFLFALSIVYIFVAPTHSTSRTALNHHHEAKVTGFQIMLEHVDSGKNLTKFELLERAIERGSRRLQRLEA MLNGPSGVETSVYAGDGEYLMNLSIGTPAQPFSAIMDTGSDLIWTQCQPCTQCFNQSTPIFNPQGSSSFSTLPCSS QLCQALPSPTCSNNFCQYTYGYGDGSETQGSMGTETLIFGSVS I PNITFGCGENNQGFGQGNGAGLVGMGRGPLSL PSQLDVTKFSYCMTPIGSSTPSTLLLGSLANSVTAGSPKTTLIQSSQIPTFYYITLSGLSVGSTRLPIDPSAFAFN SNNGTGGI I IDSGTTLTYFVDNAYQSVRQEF I SQMNLPAVNGSS S GFDLCFQMPSDP SNLQI PTFVMHFDGGDLEL PSENYFISPSNGLICLAMGSSSQGMS IFGNIQQQNMLVVYDTGNSVVSFVSAQCGAS*

\section{Nepenthesin-2, NrNep2}

MASPLYSVVLGLAIVSAIVAPTSSTSRGTLLHHGQKRPQPGLRVVLEQVDSGKNLTKYELIKRAIKRGERRMRS IN AMLQSSSGIETPVYAGDGEYLMNAAIGTPDSSFSAIMDTGSDLIWTQCAPCTQCFSQPTPIFNPQDSSSFSTLPCE SQYCQDLPSETCNNNECQYTYGYGDGSSTQGYMATETFTFETSSVPNIAFGCGQDNQGFGQGNGAGLIGMGWGPLS LPSQLGVGQFSYCMTSYGSSSPSTLALGSAAGGVPEGSPSTTLIHSSLNPTYYYITLQGITVGGDNLGIPSSTFQL QDDGTGGMI IDSGTTLTYLPQDAYNAVAQAFTDQ INLPTVDESSS GLSTCFQQPSDGSTVQVPE ISMQFDGGVLNL GEQNTLISPAEGVICLAMGSSSQLGISIFGNTQQQETQVLYDLENLAVSFVPTQCGAS*

\section{Nepenthesin-3, NrNep3}

MASSLYSFIIPLAIAFIFVTPPTSTSKISLNHEREETEPSFRIMLKRVDAGKNLTTSERIRRALQRDRKRLQMFKT MTKLSSSGIEAPVFPGPGEFLMQLAIGTPPQYYPAIMDTGSDLIWTQCSPCVLCFSQPTPFFDPSKSDSFESLSCS SKFCQDLSSACIGNCYYTYAYGDSSFTIGYMATETFAFNGSGSPVSVQNIAFGCGQFDQGTFTNCSGLVGMGRGPL SLPSQLPVSTFSYCLTSTDSSNDSTLYLGPS PYELPQGTPVTSTLVKNTYQPSFYYFTLTGISVGATRLDIPSDGF AIGKNGTNGI I ISGTTLTYIPSNYYDMAKDEFVRQTKLSPFPNSTSLIGLDCYELPQSPSTVQFPILVFHFDGGD LMVKDYLITGSYSNYELVCLAMGSSDTLGISIFGNIMQVNKYIVYDLEREVLTFIPTNCNGK*

\section{Nepenthesin-4, NrNep4}

MIMAS LLYSLLFLLGVSCVLGTSTSAAS IKS PHDVHKRDS I GLKIELRRVDS DKKYTKMERIKRAVVRGKHRHQAL TS IFSGVQTPVYSGGGEFLMNIAIGEP PNYYRAIMDTGSDLIWTQCQPCQQCFNQPTQIYNPQSSSTFSTLPCSSR YCENAWSSCDYSNYCDFNYGYGDRSSVRGYMATETFTFGTSDNSVSVPDVVFGCANDNQGTFAQFGDSGLVGFANS ALSLPYQLQTGKFAYCLTSKESTSYSTLLMGSLADQLPVA IAGTTPLISNPSRPLYYYLELQGIDVGGSPLPISSD AFA INQDGSGGMMIDSGTTYSQLPSSVMNVMANALTQITNLALVDYSSYTGLP ICMQLPTDGSSGVQLPDMVFHFE NSALYLSGEQLWTYPASGVVCLAMTSVNGLGLS I FGNSQQ INMQVVYDLQNQLLGFTPTDCDGYY*

\section{Nepenthesin-5, NrNep5}

MASSPLYSFLLELFIVCFFVAPTLATSRGAIDQGMRSPTGFRVELKHVDSGTNFTKFERIQRAVERGNHRLHKLSA MSKAVVESGVEAPVRVGQGEFLMTLAIGTPASSFSAIMDTGSDLIWTCEPCDRCFDQPTPIFLPNKSSSFSPLSCS TNLCQQLPLSTCDDGCQYVYQYGDDSSTAGVLASETFTFGSVSVLKIGFGCGSDNDGTGFSQSAGVVGLGRGPLSL VSQLDEPKFSYCLISTHEDGSKTSPLLLGSVASNLTSLKHHGA IKSTPLLHNQLQPSFYYLKLEGISVGGTRLPIN ESTFELREDGSGGFI IDSGTTITYIEESAYDVLRKEFVSQMDLTVNDNSSAGLDLCFDLPSDAQVSGELTVPELMF HFDKADLSLPGDNFMIGDDEEGLVCLAMGSSNGIS I LGNTQQQNFLVLHDLEKETVSFIPTQCSDL *

\section{Neprosin-1, NrNpr1}

MFKKASTMQAKFFTFVILSSVFYFNYPLAEARS IQARLANKPKGTIKTIKGDDGEVVDCVDIYKQPAFDHPLLKNH TLQMQPSSYASKVGEYNKLEQSWHKNGECPKGS I P IRRQVITGLPVVKKQFPNLKFAPPSANTNHQYAVIAYYYGS ASLQGANAT INIWDPTLENPNGDFSLTQIWIVAGSGSSLNTIEAGWQVYPGRTGDSQPRFF IYWTADGYTSTGCYD LTCPGFVQTNNYYAI GMALQP SMYGGQQFELNES IRMDPTTGNWWLYLWGTVVGYWPAS I YNS ITNGADTVEWGGE IYDSSGTGGFNTTTQMGSGHFPSEGYGKASYVRDLQCVDTYGNVISPSANSFQGIAPAPNCYKYQFQQGSSELFLF YGGPGCH*

7. Neprosin-2, NrNpr2 (partial) 
PFGVKAKNVSMDKLGPWHKSGKCPKGS I I KRHVNTGLPI IRKNITFRPPASYNVGHQYAVIEYMAQSQTIQGVQA TIDIWDPS LANPGGDFSLSQIWY IAGSGSDLNTAEAGWQVYPARTGDSQPRLFVYWTADAYSSTDCYDLKCLGFVQ ISGQYTHGKASTPSTDNYQQNELPLVINKDS ITEN IWLYVNGTAMGYWPNS I YNTLQGGSNIVECGGEILDSSGSG GFHTLTQMGSGYFPSAGYGLSSYIRNLLYTEDSQNWVAVTADVLQEIVPAPNCYNYSLESGQDGALWLFYGGPGCN *

8. Serine carboxypeptidase, NrSCP1 (partial)

MEKLVFLPTLFFLLQSPLSTARLLSSGVHPVPWGPKRPSSQAEALIRALNLFPREPVNIVDGPDSSWADGPRLVEK RFKFPSLDDDNGTASVEELGHHAGYYKIQDSYDARMFYFFFESRGSSNDPVVIWLTGGPGCSSELAVFYENGPFKI TDNLSLVWNEYGWDQASNI IYVDQPTGTGFSYSNDSRDIRDTEVGVSNDLYDFIQAFFAAHPDYQNNDFYITGESY AGHY I PAFAARVYQGNQANEGIQINLKGFAIGNGLTEPGIQYQAYPDFALMMGLIDQQTYDS INQTVPECENEANQ CGTNGTETCLNALSTCSTIFNS I LQVVGNINVYDIRKQCEGSLCYNFTNAQYFLNEQSVRNALGVGDIDWVPCSST VYQAMEVDWMRDLEVGIPALLDDGIRLLVYAGEYDLICNWLGNS

\section{Purple acid phosphatase, NrPAP1}

MIQSTIKYSMMDRRNPS LQVLI ITVLVLSLTSSSYSHRLYPHGHEYKIEDYDHTMIS DFRLINRMSLIKCPIESPY IQINTSATSALPNEGNITVTVSGVLHPATGDWVAMVS PSTADIGSCPLDAVMYMQTGDLASLPLLCHYPVKAQYVS NDPGYLSCQNSACQDSSCLIKTCSGSITFHYTNIRTDLIFVFFAGGVKAPCILATTQPIQFANPASPLYGHLSS ID STATSMTLTWVSGDQAPQQVLYGNNQSQTSVVTTFTQNDMCTTTLLPSPAKDFGWHDPGYIHSAVMTGLLPSTTYS YQYGSSSVGWSNQIQFRTPPAGGSNELTFLAFGDMGKTPLDPS IEHYIQPGAESVIQAMASEVAAGNIDAIYHIGD ISYATGFLVEWDFFLNLIEYIASSVSYMTS I GNHERDY INSGSVYITPDSGGECGVPYETYFPMPTPAKDKPWYS I EQGPVHFTVISTEHPWSVNSEQYNWMQS DMASVNRSRTPWLIFAGHRPMYSS IKGLEILNVDPRFVSAVEPLLLQY QVDLALFGHVHNYERLCAVYQKQCLGMPVKDANGIDTYDNGNYTAPVHVI IGMAGFTLDSFTSNPSSWSLVRISEF GYSKVQATPNSLLFQFVNAHTRQIDDSFYITK*

\section{S-like ribonuclease, NrRNases}

MKYCNFYSVFVKLLILQSLAVVVRCDYDFFYFVQQWPGSYCDTSEGCCYPTTGKPATDFGIHGLWPNNDDGSYPSN CDPNNPFDQSQIQDLLSSLQAEWPSLSCPSSNGISFWTHEWDKHGTCSESVLDEHDYFAAALALKSQANLLQSLEN AGINPNGSFYALSDVDNAIAQATGNTNYGYVSCNTDDYGNGQLYQVYICVDHSGQNF INCPTNGVTDQNCPSSVEF $\mathrm{PSF}^{*}$

\section{Endonuclease 2, NrEndo2}

MRSNRRPLPLLASLCVLFVGIPGCHGWGWDGHYTTCKLAEPYYSNATVAAIQNLLAPGQYLPDLCSWADEVRSVYK WSAALHFADVPDDSCVFNYTRDCHDDYGVPGRCVVGA IYNYTTQLI DGYSS GNSPYNLTQALLFLSHFMGDI HQPL HLGHEGDRGGNS I IVYWYNTRTNLHHVWDTSMIYTEEDDFYNGSVDQLEAAIAQNITSDEVQQWQTCSGGQPPCPI VYASESVPLACEWAYNGVTNYSTLGDDYFYSRWPIVQQQLSRGGVRLAAALNRIFGSS *

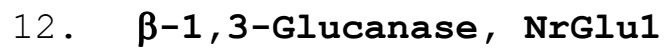

MFIALLLGILFATINTRASQIGTCFGTLGDNLPSRPQVVAQYNQYNIERMRIYSPDSSLSQALSGSGIELMLGILN QDLQPIASSQSNANSWVQDNIGAYPNVNFRYLAVGNE IRPNLNNGAAQYAQYVLPAMQNLQNAINQMGYGGRIKVS TAMEMGIAINTYPPSAGQFDPS ISDY INPIVSFMRDNGS PLLLNCYPYFAYAYSSNIDLSYALFTSPGTVVQDGQY AYQNLFDAMVDS IYSALEKAGCGSVVIVVSESGWPTMGGKGTS I DNAKTYNNNLIQNVKKGTPKRPGAYLETYIFD MYDEDLKSSELEQHWGLFTANGDLKYPVNFN*

\section{Acidic endochitinase, NrChit3}

MKTHYSSA I LPIVTLLVFLS INPSHGSGIAVYWGQNGNEGTLSDTCATGNYQYVLISFLTTFGNGQTPVLNLAGHC DPSSNGCTGLSTDITSCQNQGIKVLLSLGGASGSYSLVSTDDADQVAAYLWNNYLGGQSDSRPLGSAVLDGIDFDI ESGSDNYWGDLASALKNYSQSVLVSAAPQCPYPDAHLDLAIATGIFDYVWVQFYNNEQCEYVNDDTNLLSAWNQWT SSQANVVFLGLPASTDAASSGYISPDVLISQVLPS IKASSKYGGVMLWSKYYDNGYSSAIKDSV*

\section{Chitinase, NrChit1}


MALSTEINLLTLAFLAGILAGVLPILVSGQNCGCAANLCCSKWGYCGTGDAYCGPGCQEGPCYSSGGRGGGSSVAD IVTDSFFDGI INQASS SCAGKYFYSRSAFLDALDSYPGFGTSSDADTNKREIAAFFAHVTHETGHFCYIEEIGGSS LPTSAYCDPSAVQWPCNPDVGYYGRGPIQISWNYNYGPAGQA I GFDGLNSPQTVANDPT ISFKSALWFWMKNVHS I IVSGQGFGATIRAINSMECNGGNPSAVDDRVGYYVAYCNQFGVSPGGDLYC*

\section{DOMON-like domain, NrDom1}

MASLQFSS IVLCFAFVFLLSPSAYSQTCKSQKFTGQKKFETCTDLPHLNASLHWTFDSSESSLS IAYIAPPAASDG WVAWA INPTSVGMIGAQALIAFKQPNGSLTVETFDLKSYQSIVQGP ISYTVSGTSAEYSGGNITIFATWALPAKTT QVNQVWQVGSTVVNGVPQRHALQQDNLLSKGVLTLVETAAAPAGSPTGSSGSGGSQAESPTGSSGSGGSQAESPTG SSGSGASPAPTGGNSGAGESTMRTKNEWVCGVLTFTAAFALLF*

\section{Thaumatin-like Protein, NrTLP1}

MSHFTKFI IFSSLLLALFSVS I DAATFVITNNCPYTVWAAAS PGGGQQLDPGQTWTINVNAGQTGGRVWARTGCNL SGSNGARCQTGDCGGLLQCQGYGSPPNTLAEYALNQYMNLDFFDISLVDGFNVPLSFLPTSNGCTNGPTCTADVNG RCPSVLKAPGGCNNPCTVFKTNEYCCNSGSCGPTDYSKYFKGLCPNAYSYPKDDATSTYTCPGGTNYKVVFCP*

\section{Hevein, NrHev1}

MGRENLWAALIMLCLAAGAAAQQCGRQAGGALCPNNLCCSQWGYCGSTDQYCS PAQNCQSNCKGGGGGSSPAGGGT GEGANNVRATYHLYNPQDHGWSLYAVSAYCSTWDGDKPLAWRSKYGWTAFCGPVGPHGQAACGKCLKVTNVATGAE TTVRIVDQCSNGGLDLDVNVFKKLDTNGQGYAQGHLTVNYQFVDCGD*

\section{Cationic peroxidase $1, \operatorname{NrPrx} 1$}

MATLSFLPLCLVWLVLLGAASAQLSSTYYDSSCPKALST IESGVISAIQKETRMGASLLRLHFHDCFVNGCDGSVL LDDTANFTGEKTAAPNLNSLRGFNVIDTIKASVEKVCPGVVSCADI LAVVARDSVVRLGGQSWTVLLGRRDSTTAS LSAANADI PAPTLNLNGLISSFSNKGLTEDEMVALSGAHTIGLARCVTFRSRIYNETNIKSSYAASLKKICPTSGG GNNTAPLDITSPFIFDNAYFKDLINLEGLLHSDQQLYNNGSADSQVSKYSSSPSTFSTDFGNAMIKMGNLSPLTGS EGQIRTNCRKVNP*

\section{Cationic peroxidase 2, NrPrx2}

MASSRSLRLCLVWLALFGTASAQLSSTYYDSSCPNALSTIKSGVTSAINNEARMGASLLRLHFHDCFVNGCDASVL LDDTANFTGEKTALPNLNSLRGFTVIDTIKASVESVCPGVVSCADI LAVAARDSVVALGGPSWTVQLGRRDSTTAS LSDANSDI PAPTLNLSGLITSFSNQGFTEDEMVALSGAHTIGLARCTTFRSRIYNETNIDSSYAASLQAICPSSGG DNNTAPLDTTSSTTFDNAYYTNLISLEGLLHSDQQLYNNGSADSQVSNYSSNSSTFFTDFANAMIKMGNLSPLTGS EGEIRTNCSLINS*

\section{Peroxidase $3, \operatorname{NrPrx} 3$}

MTALKLSVLFFLELVIQLAIVANSQELVLGFYNDTCPEAENIVNQVTALYIYNDSTLAPALLRLTFHDCFVRGCDA SILLEPTNANNATEITAPPNLTLRGFKVIDAIKTKLEEYCPGVVSCADI IQLAGRDAIQTAYGASWVVLTGRRDGT VSLASEALEDLPSPYSNFDTLKQKFEAVGLSVKDLVVLSAAHTVGVAHCPSFSGRLYNFSGEGGTDPSLDPNYAKF LKQKCPPNDQLTTVYQDPITPNKFDIGYYVAVDQHEGLFESDAALLDDNETRGYLESQLWTMGWTWLEDFGVSMMK MYKEKVLTGTEGEIRKKCGYVN*

\section{Leu-rich repeat receptor-like protein kinase, NrRPK1}

MKASDLKSLFVLLCLIAS IADCEDDVRCLEGLKS S LEDPEGKLS DWTFANTTIGY ICKYVGVSCWNDLENRVNS LE LPDSELGGQ I PDAFQYCGSLQTLDLSGNSLSGT I PSQ ICTWLPFLVFLDLSNNQLSGQI PPDLGNCKYLNKLSLAD NQLSGNI PYQLSNLIRLNSFSVANNHLTGRI LDSLSHFDKSDFEGNDGLCGKPARKCGGLSKKNLAI I IAAGVFGA AASLLLGFGLWWWYFSRASQRRRHGYGLGRADDGSWIDRLRAQKLVQVSLFQKPLVKLRLADLLAATNNFSQENVV ISNRTGATYKAMLSDGSALAIKRLNTCKLSEKQFRGE INRLGNLRH PNLVPLLGFC IVEEEKLLVFKHMSNGTLYS MLHGATVLDWPTRFRISLGLARGLAWLHHGNQP PYLHQS ISSNVILLDEDFDARI I DFGLARLMNASDPNESSFVN GELGEFGYIAPEYSSTMTVSLKGDVYAFGVVLLELVTGQKPLEVNTNEEGFKGNLVDWVNQLTNSGRVDEAIDKDL SGSGHNDE IHQVLRIACNCVVSQPKDRFSMYQAYESLKTVANDHGSSEQYDEFPLIFGGQDTDNTV* 


\section{Leu-rich repeat protein, NrLRR1}

MLYGEPPNFLFYQHDLRVADLSDSNLQGNFPAWLFDNNTRLQGLHLRQSLLTGPFQFPVHSHLS IATIDVSHNEMV GKI PVNFNVTFPNLKRLDMSNNAFERKSPPCFRDMNSLQTLNLSNNHLSGELPQNFVAGSP IQFLDISNNDLEGP I SWVSNLTHLDSLYLQGNNFS GKI PVNLSTNLQTMDLSSNHFS GELPRWMNVSNFKE INMSQNHLEGQ I PEEYCELY NLDFLDLSENNLTGI I P PCFPPFIKYVHLSKNRLSGPFPYAFRSNTDLLVLDLSYNNLTGRLPNWIGNLGKLS ILL LGSNHFEGNIPTGLCNLNQLS I LDLSKNNLYGP I P PCLNNITFQPSDAKAYDGSSFTLSEQPLYEKTKGKMPYLDD TNYTMIYNDLFLS IQERVEFTTKS IAYSYEGKVLVYMSG IDLSCNKLTGEIPPDMGSLSEIRALNLSHNNLSGS IP ATFSNLGQIESLDLSYNFLNGS IPPELTQLTSLEAFSVAHNNLSGMTLGLKDQFGTFGEASYEGNPFLCGPPLPKN CTDAGSPSLIP ISEEDDQEDNDDDW I DMGTFYVSFVVSYI IMFLGIVIVLVINPNWRRRWFCLVDNIVISSYCFVV VNFRKLKKC *

\section{ADP/ATP carrier protein, NrADT1 (partial)}

SKTAAAPIERVKLLIQNQDEMIKAGRLDRRYTGIGECFKRTAADEGVLSLWRGNTANVIRYFPTQALNFAFRDKFK KMFGFKKDRDGYGMWMLGNLASGGAAGATSMLFVYSLDYARTRLANDAKSAKKGGERQFNGLVDVYRKTLASDGIA GLYRGFMPSVAGI IVYRGLYFGMYDS I KPVLLVGSLANNFLASFALGWCVTTGAGIAAYPLDT IRRRMMMTSGEAV KYKNSFDAARQI IAKNGVKSLFNGAGANILRGVAGAGVLS IYDQLQVLLFGKAFSGGSG*

\section{Glyceraldehyde-3-phosphate dehydrogenase, NrGPD1}

MGKIKVGINGFGRIGRLVARVILQSEDVELVAVNDP F STDYMTYMFKYDSVHGQWKHHDVKVKDDKTLLFGEKSV TVFGIRNPEEIPWGETGADYVVESTGVFTDKDKAAAHLKGGAKKVVISAPSKDAPMFVVGVNEKEYKSELDIVSNA SCTTNCLAPLAKVIHDRFGIVEGLMTTVHSITATQKTVDGPSMKDWRGGRAAS FNI IPSSTGAAKAVGKVLPALNG KLTGMAFRVPT I DVSVVDLTVRLEKAASYTEIKAAIKEESETKLKGI LGY IEHDAVSTDF I GDSRSS IFDAKAGIA LNDNYVKLVAWYDNEWGYSSRVVDLIRHIASTK*

\section{ATP synthase subunit alpha, NrATPA (partial)}

MALSLSEISVLLEQKISNSYSKINIDEIGRVLSVGDGIARVYGLNKIQAGEMVEFASGVRGMALNLENDNVGIVLF GPDNA INEGDIVKRTGT IVDVPVGKGTLGRVLDALGNP I DGKGPLKDVTRQRAEVKAPGI IARQSVNEPMQTGLKA VDSLVPIGRGQRELIIGD

\section{ATP synthase subunit beta, NrATPB (partial)}

MS ISTELKNIGRITQI I GPVLDVSEPPGKMPNIYNALTVSGKNEAGQEIALTCEVQQLLGDHCVRAVAMSATDGLM RGMDVIDSGRPLSVPVGQSTLGRI FNVLGEPVDNLGPVNTKEQLP I HRSAPAFVDLDTKLS I FETGIKVVDLLAPY RRGGKIGLFGGAGVGKTVLIMELINNIAKAHGGVSVFGGVGERTREGNDLYMEMKESGVINESNIAESKVALVYGQ MNEP PGARMRVGLTALTMAEYFRDINKQDVLLFIDNI FRFVQAGSEVSALLGRMP SAVGYQPTLATEMGGLQERIT STKDGSITSIQAVYVPADDLTDPAPATTFAHLDATTVLSRNLASKGIYPAVDPLDSTSTMLQPWIVGDEHYQCAQN VKQTLQRYKELQDI IAILGLDELSEEDRLVVARARKIERFLSQPFFVAEVFTGSPGKYVSLAETIQGFTLILSGEL DDLPEQAFYLVGNIDEAV

\section{Enolase, NrEno1 (partial)}

EVYHVLKGVIKKKYGQDATNVGDEGGFAPNIQENKEGLELLKTAIDKAGYTGKVVIGMDVAASEFYKEDKTYDLNF KEENNDGSQKISGDALIDLYKSFVSEYPIVSIEDPFDQDDWEHYTKLTAEIGDQVQIVGDDLLVTNPKRVEKAIKS KACNALLLKVNQI GSVTES IEAVKMSKHAGWGVMASHRSGETEDTF IADLSVGLATGQIKTGAPCRSERLAKYNQL LRIEEELGDGAVYAGKNFRQPVEPY*

\section{Actin, NrAct1}

MADAEEIQPLVCDNGTGMVKAGFAGDDAPRAVFPS IVGRPRHTGVMVGMGQKDAYVGDEAQSKRGILTLKYPIEHG IVSNWDDMEKIWHHTFYNELRVAPEEHPVLLTEAPLNPKANREKMTQIMFETFNVPAMYVAIQAVLSLYASGRTTG IVLDSGDGVSHTVP IYEGYALPHAI LRLDLAGRDLTDALMKILTERGYMFTTTAEREIVRDIKEKLAYVALDYEQE 
LETAKSSSS IEKNYELPDGQVITIGAERFRCPEVLFQPSLIGMEAAG I HETTYNS IMKCDVDIRKDLYGNIVLSGG STMFPGIADRMSKEITALAPSSMKIKVVAPPERKYSVWIGGS ILASLSTFQQMWISKGEYDESGPSIVHRKCF*

\section{Tic20-like protein, NrTic20}

MIVNGWTITSGTMQ INSGACKPVSYNPTCPIATLLPPRAALPDVRSSYGLHRELKSSLSRGTSFLHLSAASSLLLN GEQGGLLNTIPWLPKRRRHVTPPRASKDVPYSYRYP PMTKKPRWWWRTLSCLPYLMPLHETWMYAETAYHLHPFLE DFEFLTYPFLGAIGSLPSWFLMAYFFVAYLGIVRRKEWPHFFRFHVVMGMLLEIALQVIGTVSRWMPLGVYWGKIG MHFWTAVAFGYLFTVLESIRCALAGMYADIPFVCDAAYIQIPYD*

\section{Protein transporter, NrSec1}

MVQALPQYQEQVEKLS I HVEIAGKINQI IRDVGLRDLGQLEQDLVFGDAGAKEVINFLRTNQDAPSENKLRLLMIY ASVHP DKFEGDKASKLMQLAKLSSKDMKAVQNMRLLAGSRDTQKKSNGSFSLKFDAQKKKVAARKDRAHADEETWA LSRFYPMLEELIENLSKGELPKNGYRCLNEPSPSATEDGQNLSVRKNSTI PRSMRSRRTATWAQPHHSDDGHGSDS ILGNASNDLKKMGPRIFIFI IGGATRSELRVCHKLTTKLRREIVLGSTSMDDP PQYITKLKLLSEEELSLDGIKL*

\section{Elongation factor 1-alpha, NrEF1a}

MGKEKVHINIVVIGHVDSGKSTTTGHLIYKLGGIDKRVIERFEKEAAEMNKRSFKYAWVLDKLKAERERGITIDIA LWKFETTKYYCTVIDAPGHRDF I KNMITGTSQADCAVLI IDSTTGGFEAGISKDGQTREHALLAFTLGVKQMICCC NKMDATTPKYSRARYDEIVKEVSSYLKKVGYNPEKI PFVPISGFEGDNMIERSTNLDWYKGPTLLDALDLIQEPKR PSDKPLRLPLQDVYKIGGIGTVPVGRVETGVLKPGMVVTFGPTGLTTEVKSVEMHHEALQEALPGDNVGFNVKNVA VKDLKRGFVASNSKDDPAKEAANFTSQVI IMNHPGQI GNGYAPVLDCHTSH IAVKFSEILTKIDRRSGKELEKEPK FLKNGDAGFVKMIPTKPMVVETFSEYPPLGRFAVRDMRQTVAVGVIKSVEKKEPTGAKVTKAAAKKGAK*

\section{Elongation factor 2, NrEF2}

MVNFTVDEIRALMDKKKNIRNMSVIAHVDHGKSTLTDSLVCKAGIIAQQKAGEMRFTDTRKDEQERCITIKSTAIS LYYELPSKDLGFIKQEREPEISHFLINLIDSPGHVDFSSEVTAALRVTDGALVVVDCVSGVCVQTETVLRQAIAER IKP I LFMNKMDRALLELQLQQEDLFQTFQRIVENVNVI IATYGDDSGPMGELQVDPTKGTVGFGAGLHGWAFTLKE FAEMYASKFKIEVDKLMKRLWGDNFFSPSEKKWSKTGGEGYNRGFCQFVLDPIFKVFRA IMDCKKDEYIALLDKLS IKLQGDDRDKLEEGGKPLLKLVMKQWLPAGDVLLTMIAIHLPS PVVAQKYRAELLYEGPQDDEAFLGIKTCDPTGP LMMY I SKMVPT SDKGRFYAFGRVFSGVVQTGQKARIMGPNYVPGKKEDLYVKS IQRT I LMMGRYTEP IEDVPCGN I CGLVGVDQYLVKTGTITTFENAHNLRVMKFSVS PVVRVAVEPKNPADLPKLVEGLKRLAKSDPMVQCI IEESGEH I VAGAGELHLEICLKDLEEDHAC I P IKVSDPVVSYRETVSEESE IMCLSKS PNKHNRIFLKARPMP DGLPEDIDKGD VTPRQEFKARARYLNEKYEYDVNEARKIWCFGPEGTGPNLLMDCTKGVQYLNEIKDSCVAGFQWATKEGVLAEENV RGVRFDI HDVTLHADA I HRGGGQI I PTARRVLYASMLTAKPRLFEPVYLCEVQCPEVAVGG IYGVLNRRRGHVFEE HQVTGTPMF IVKAYLPVNESFGFTADLRSNTGGQAFPQCVFDHWQVMNQDPFDATTKIRQIVNDIRKRKGLKEGIP PLDDYYDKL*

\section{Ribosomal protein $\mathrm{S14}$, NrRS14}

MSKRKTREPKEENVNLGPAVREGEHVFGVAHIFASFNDTF I HVTDLSGRETLVRVTGGMKVKADRDESSPYAAMLA AQDVSQRCKELGITALH IKLRATGGNKTKTPGPGAQSALRALARS GMKIGRIEDVTP I PTDSTRRKGGRRGRRL *

\section{Chaperone protein, NrHsp90}

MAS I LVRS LA IVSLASFPSSSTAVKNS HNKVFSLKS S FLPQNYGTNRI PMYGKRCS IRLERRANRLAVRCEAAVAE KEAPETEGEKFEYQAEVSRLLDLIVHSLYSHKEVFLRELVSNASDALDKLRFLSVTEPS LLGDASELEIRIKADPD NGTITIIDTGVGMTKEELVDCLGTIAQSGTSKFLKALKENKDVGADNGLIGQFGVGFYSAFLVAERVVVSTKSPRS DKQFVWEAVADSSSYTIREETDPEKLSKRGTQVTLYLRADDKYEFTDSTKIQNLVKNYSQFVSFPIYTWQEKSRTV EVEEEQPKEGEEEKPEAEKTKKKKTVTEKYWDWELANETKPIWMRSAKDVQKEEYDEFYKKTFNEFLDPLAHTHFT TEGEVEFRSVLYI PGMAPLNNEDIVNPKTKNIRLYVKRVFISDDFDGELFPRYLSFVKGVVDSDDLPLNVSREILQ ESRIVRIMRKRLVRKTFDMIQE ISENENKEDYKKFWENFGKFLKLGCIEDSGNHKRLTPLLRFYSSKSEEELISLD 
DYVENMGENHKAIYYLATDSLKSAKTAPFLEKLLQKDIEVLYLIEPIDEVAIQNLQTYKEKKFVDVSKEDLELGDE DEVKERETKQEYNLVCDWIKQQLGEKVAKVQVSKRLSSS PCVLVSGKFGWSANMERLMKAQTLGDTSSLEFMRGRR ILEINPDHP I IKDLSAACKNAPDSNDAKKAVELLYDTALVSSGFS P DSPAELGTKIYEMMTMALGGRWGRIEEGSD ALEDNSPESDASADEASDAQVVEPSVIEPSEVRTDEHDPWQD*

35. Histone acetyltransferase, NrHAC1 (partial)

MASVGNQFPQIGGGGLGLMSPSGFRFNLRHEDPCRGYVRTTIAKFLTQNSSGNYEQQIAELAADLENHLFTDAVCK EDYLNRESFYDRLQNLIQKKWLHSKFAQQEVFRCPSSFLPIATDALAPSANLLDPFP DNAMA IGTGCNVVGPDDVN QPQVFADVSLNSLSGSQTKYNKHGVAASSLGGQCAFGQTSTAHEQVMS DNYTPKEVFSYFKPVDSGRTLSQS I LLY DSETSFESNHGSQHIADGTGLIMSTRKNCPLIDVPSLGWKKSKLETVGVSDGEFLNISSTTGKVPMVESQQLLPNL QFVKP FVEGESCSQFSLKHKDSRA I HPDASGNFPLHSVSNGSGQCSVLNNLS QNLVKE I LCCY IRYNSCPSPVGKS LVSFLEYVHSKKCTGFKCRCEQYNSLLYHFDNCHSSDCDICSPALKLHLMDKAGSEIFRSGN IWMSYGRNHSWRGT SCAAKDYPTPAKRRKADISSKNGLSSSFSPLIDHSLSP DGPLNLQQLSGSPVSSFSEATELKMEAVSVHTWNSASS KETRKMT INNPLRFHYEDMYNPSEEPSVDYKQMEMETASNKEVKDKFTENFGTMS LECSS I LSEEQHIAETEGLNI RTKSGEIDPDITREFMEPLSASGPEMKSEKQNVKGVSLTEFFTAEEVKQHLWSLRQSASEEVFGNITSHSSNVNTC QLCALDKLAFQSMP IYCSACSARIKSHVTYYSMLDDSGAQLCFCSSCCKKSRGGNITFFGLSVPKSKLEMKKNDVI TEESWVQCDKC

36. Auxin response factor, NrARF1 (partial)

AWDEPDLLQNVKRVNPWLVEPVASMPSVHMSPFSSPRKKLRLPQHPDFPFADQLPLISPFSSNTFRPSSPLCCISG NTSAGIQGARHARLGQSLSDLH INKLQSSLFGTHPRFSGPSFTCKP IMNEDISCLLTMGSRNQSTKKNDDVKTPKF VLFGKPILTEEQISQSSSGDSLGNSSSDRNLEKMANLSDGCNGLMDNSSDGFFPICRDGLKIKLGLETGHCKFF* 